\title{
Image Sharpness Measurement in Scanning Electron Microscopy Based On Derivative Method In ISO/TS 24597 document
}

\author{
Sunghyon Kim ${ }^{1}$, Byong Chon Park ${ }^{2}$, Il-seok $\mathrm{Oh}^{1}$ and Jin Seung Kim ${ }^{1}$ \\ 1. Department of Nano Science and Technology, Chonbuk National University, Jeonju, Rep. of Korea. \\ 2. Center for Nanometrology, Korea Research Institute of Standards and Science, Daejeon, Rep. of \\ Korea.
}

Resolution is a key performance metric of SEM. Generally an image of gold-on-carbon sample is taken, and the image sharpness is determined either manually or with a computer program. Computer-based resolution-calculation methods are more objective, while it should deal with the actual complicated SEM images in the less-smart manner as the humans. Further the algorithm may need compromise between effectiveness and precision, and come in a variety of versions. The compatibility of the programs should be checked with a standard SEM image, whether it is real or simulated. It will be further worth to standardize the algorithm itself, otherwise at least to develop the inspection methods to test the algorithms.

Algorithm to measure SEM image sharpness has been developed basically according to the derivative method as stated in ISO/TS 24597 document [1], and implemented in a MATLAB-based program. There are some replacements of the subroutines or addition of algorithms, but these adaptations are minor. Out of the obtained sharpness, four-standard deviation outliers are removed, and the mean of the remained values are reported as the final sharpness. The results are displayed in polar plots to reveal the anisotropic noise characteristics as well as the astigmatism of the optics. Throughout the calculations, the intensity profile of the electron probe is assumed to be Gaussian with the $1 / \mathrm{e}^{2}$ radius of sigma.

The accuracy of the algorithm was first examined using a set of the numerically generated circular reference images. As the edge curve extends over the edges of the reference circle reasonably well, the image sharpness calculation proved to be perfect. Fig.1 illustrates an example for the case of the sigma $=2$ pixels. Further the robustness and compatibility was tested using the five state-of-the-art field emission SEM images. The images were taken with either of the acceleration voltages, $1 \mathrm{kV}$ and $15 \mathrm{kV}$. The gold-on-carbon samples and the machines are all different. Figure 2 and Figure 3 illustrates the two examples acquired with the acceleration voltages of 15 and $1 \mathrm{kV}$, respectively.

We conclude that the calculated sharpness coincides with those of the artificial images, and appears to be compatible with the existing programs. We are currently collecting more extensive data while improving our algorithm. [4]

\section{References:}

[1] Microbeam analysis - Scanning electron microscopy - Methods of evaluating image sharpness, ISO/TS 24597:2011(E).

[2] P. Cizmar et al, Scanning 30, 1 (2008).

[3] B. Rieger, G.N.A. van Veen, EMC 2008 Vol 1: Instrumentation and Methods, p. 613 (2008).

[4] This work was supported in part by the KRISS program (Grant No. 15202009) and the IT R\&D program of the MKE/KEIT (Grant No. 15201006 ). 


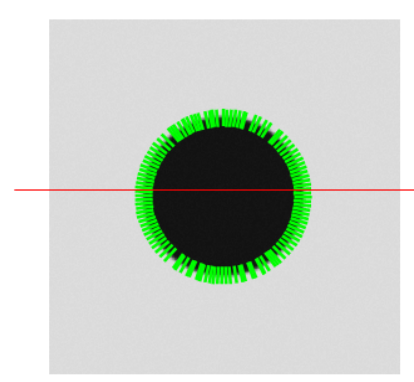

(a)

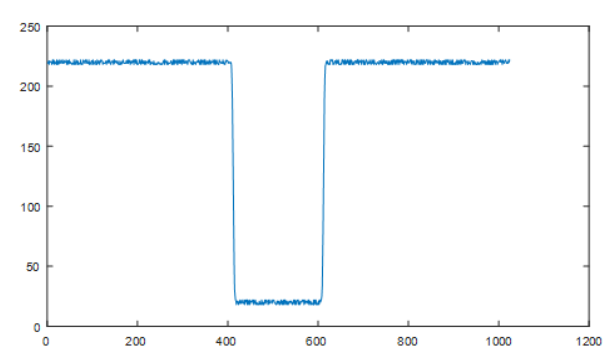

(b)

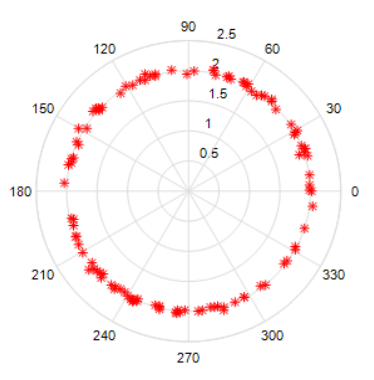

(c)

Figure 1. (a) The reference circle image. Green lines and a red line represent the edge segments and the position of the cross-sectional view shown in (b). (c) polar plot of the sigmas from the fit of the error function to the edge intensity profiles. The polar angle around the outer circle (unit=degree) represents the direction of the edge segments. The number radially placed represents the sigma in terms of pixel.

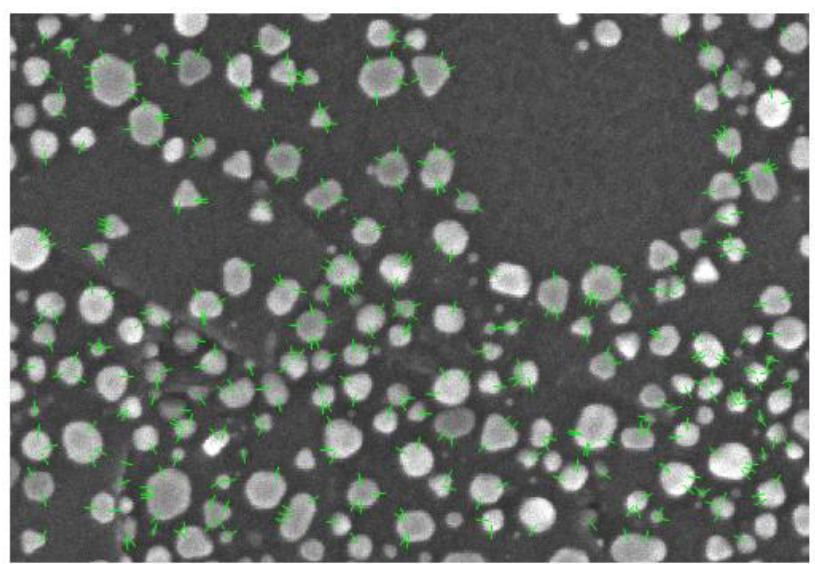

(a)

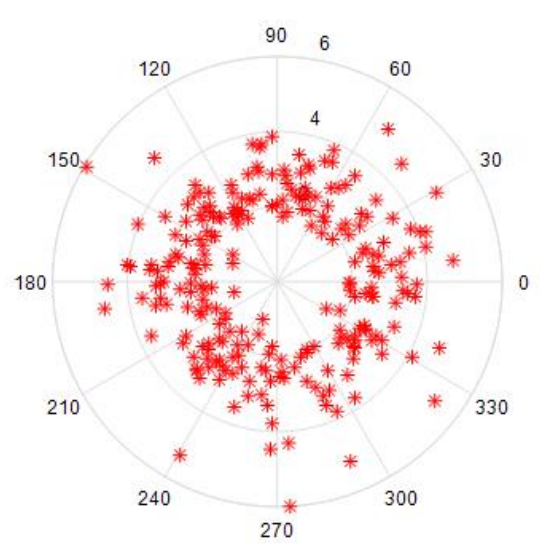

(b)

Figure 2. (a) The $15 \mathrm{kV}-\mathrm{SEM}$ image of a gold-on-carbon sample overlaid with edge segments (370 lines, green). (b) is the polar plot of the obtained sigmas from the fit of the error function to the intensity profiles for the edge segments.

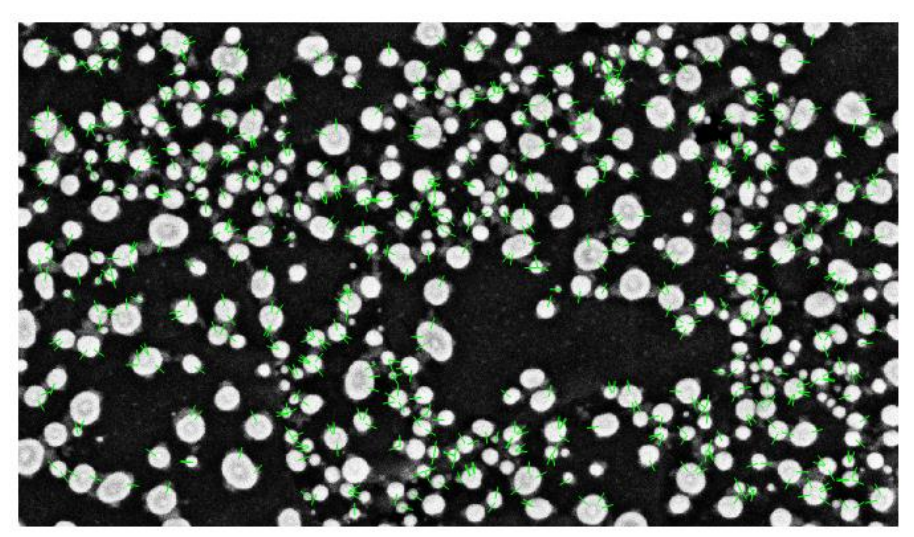

(a)

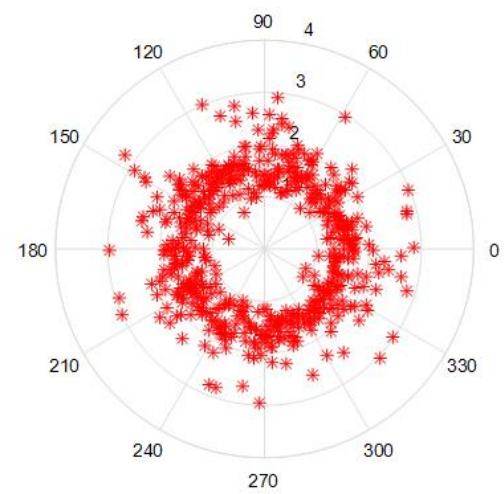

(b)

Figure 3. (a) The $1 \mathrm{kV}$-SEM image of a gold-on-carbon sample overlaid with edge line segments (660 lines, green). (b) is the polar plot of the obtained sigmas from the fit of the error function to the intensity profiles for edge segments. 